\title{
Relapse of Nephrotic Syndrome after Adrenocorticotropic Hormone-Induced Remission: Implications of Adrenocorticotropic Hormone Antibodies
}

\author{
Snehal Shrivastava Bohan Chen Lance D. Dworkin Deepak K. Malhotra \\ Rujun Gong
}

Division of Nephrology, Department of Medicine, University of Toledo College of Medicine, Toledo, OH, USA

\author{
Keywords \\ Corticotropin · Focal segmental glomerulosclerosis . \\ Proteinuria $\cdot$ Neutralizing antibody
}

\begin{abstract}
Background: Prolonged use of corticosteroids continues to be the mainstay in the management of most proteinuric glomerulopathies, but is limited by extensive side effects. Alternative medications such as adrenocorticotropic hormone (ACTH) have been recently used to treat refractory glomerulopathies and have shown superior outcomes when compared with steroids. However, the clinical responsiveness to ACTH therapy varies considerably with a number of patients exhibiting de novo or acquired resistance. The underlying mechanism remains unknown. Methods: A patient with steroid-dependent focal segmental glomerulosclerosis (FSGS) developed severe steroid side effects impacting quality of life and was converted to repository porcine ACTH therapy. Immediate response in the form of remission of nephrotic syndrome was noted followed by relapse in 10 weeks. Suspecting the role of some ACTH-antagonizing factors, the patient's serum was examined. Results: Immunoblot-based antibody assay revealed high titers of de novo IgG antibodies in the patient's serum that were reactive to the porcine corticotropin with negligible cross-reactivity to human corticotropin.
\end{abstract}

In vitro, in cultured B16 melanoma cells that express abundant melanocortin receptors, addition of the patient's serum substantially abrogated the porcine corticotropin triggered signaling activity of the melanocortinergic pathway, marked by phosphorylation of glycogen synthase kinase $3 \beta$, thus suggesting a mitigating effect on the biological functionality of porcine corticotropin. Conclusion: ACTH is a useful alternative therapeutic modality for refractory proteinuric glomerulopathies like FSGS. However, as quintessential therapeutic biologics, natural ACTH, regardless of purity and origin, is inevitably antigenic and may cause the formation of neutralizing antibodies in some sensitive patients, followed by resistance to ACTH therapy. It is imperative to develop ACTH analogues with less immunogenicity for improving its responsiveness in patients with glomerular diseases.

(c) 2020 S. Karger AG, Basel

\section{Background}

Treatment of relapsing nephrotic syndrome is challenging despite the advent of newer immunosuppressive medications. Prolonged use of glucocorticoids continues

S.S. and B.C. contributed equally to this work.

\section{KARGER}

(C) 2020 S. Karger AG, Basel

karger@karger.com

www.karger.com/ajn
Dr. Rujun Gong

Division of Nephrology, Department of Medicine

University of Toledo Medical Center

3000 Arlington Avenue, Toledo, OH 43614 (USA)

E-Mail Rujun.Gong@UToledo.edu 

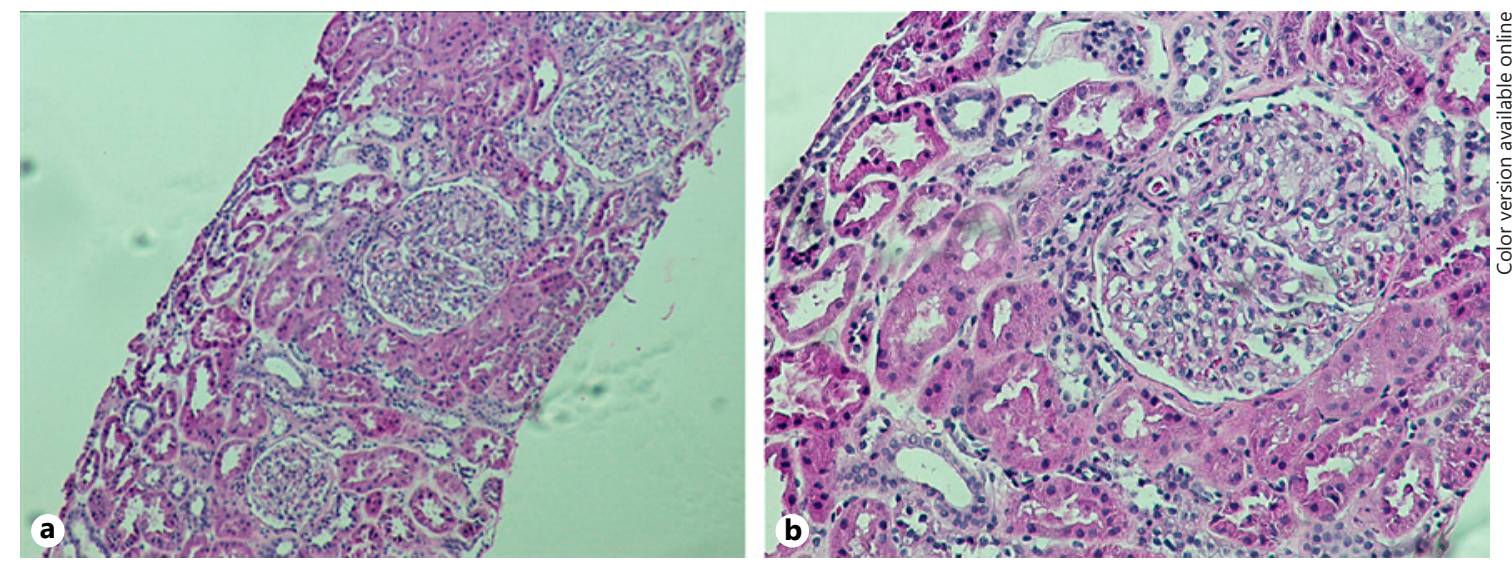

Fig. 1. Histology of kidney biopsy. The patient's kidney biopsy specimens were processed for routine periodic acid-Schiff staining. a Low power view shows glomerular size variability suggesting glomerulomegaly, a possibly early sign of FSGS, with otherwise normal appearance. $\mathbf{b}$ High power view shows near normal morphology of glomerulus. to be the mainstay in management of proteinuric glomerulopathies. The extensive side-effect profile of glucocorticoids limits their use for prolonged therapy. Alternative medications such as animal-derived adrenocorticotropic hormone $(\mathrm{ACTH})$ have been recently used in pediatric and adult patients to treat resistant diseases, such as minimal change disease (MCD), focal segmental glomerulosclerosis (FSGS), membranoproliferative glomerulonephritis, and others. ACTH has shown to induce remission of nephrotic syndrome not amenable to steroids and other immunosuppression medications. A multicenter small randomized controlled trial in patients with idiopathic membranous nephropathy demonstrated superior outcomes with ACTH when compared with the standard Ponticelli regimen [1]. It is hypothesized that the antiproteinuric action of ACTH is not solely attributed to its steroidogenic effects because a number of case series studies found that patients with steroid-resistant nephrotic syndrome still respond well to ACTH therapy [2]. Increasing evidence suggests that ACTH may exert a glomerular protective and antiproteinuric effect via multipronged mechanisms, including direct podocyte protection, lipid lowering action, and potent melanocortinergic activities [3].

The clinical responsiveness to ACTH therapy varies, however, considerably with a number of patients exhibiting resistance to treatment $[4,5]$. For instance, in a study in patients with idiopathic FSGS, only 7 out of 24 patients responded to ACTH therapy and achieved clinical remission [4]. In another study to determine the effectiveness of ACTH therapy in idiopathic membranous nephropathy, 8 out of 20 patients failed to achieve proteinuria remission after ACTH gel treatment for 1 year [5]. The underlying mechanism remains unknown. As quintessential therapeutic biologics, animal-derived natural ACTH, similar to other biologics like insulin and erythropoietin, regardless of purity and origin, is inevitably antigenic, and thus may have drawbacks like treatment resistance [6]. Antibody development against therapeutic animal-derived ACTH has been reported decades ago in patients with rheumatologic disorders [7-9]. Lately, with the renaissance of ACTH therapy in proteinuric glomerulopathies, de novo formation of antibodies against short-acting ACTH of mixed bovine, ovine, and porcine origins has been noted in a pediatric patient [10]. Here, we present the first proof of concept to measure antibodies against long-acting porcine ACTH in an adult patient after initial complete remission.

\section{Case Description}

A 25-year-old woman of Asian origin was followed at the $\mathrm{Ne}$ phrology Clinic of the University of Toledo Medical Center for the management of her nephrotic syndrome. She initially presented with symptoms of foamy urine, fatigue and lower extremity swelling at the age of 17 years. Laboratory evaluation showed proteinuria of $5 \mathrm{~g} / 24 \mathrm{~h}$ and urine analysis was positive for proteinuria, hematuria, and no casts were noted. She underwent renal biopsy that showed MCD and early signs of FSGS (Fig. 1a, b).

At the time of presentation and throughout the disease course, her renal function was preserved as evaluated by the measurement of serum creatinine and by estimated glomerular filtration rate. She had been normotensive with blood pressure readings in the range of $120 / 60 \mathrm{~mm} \mathrm{Hg}$ and no other significant personal or family medical history. 


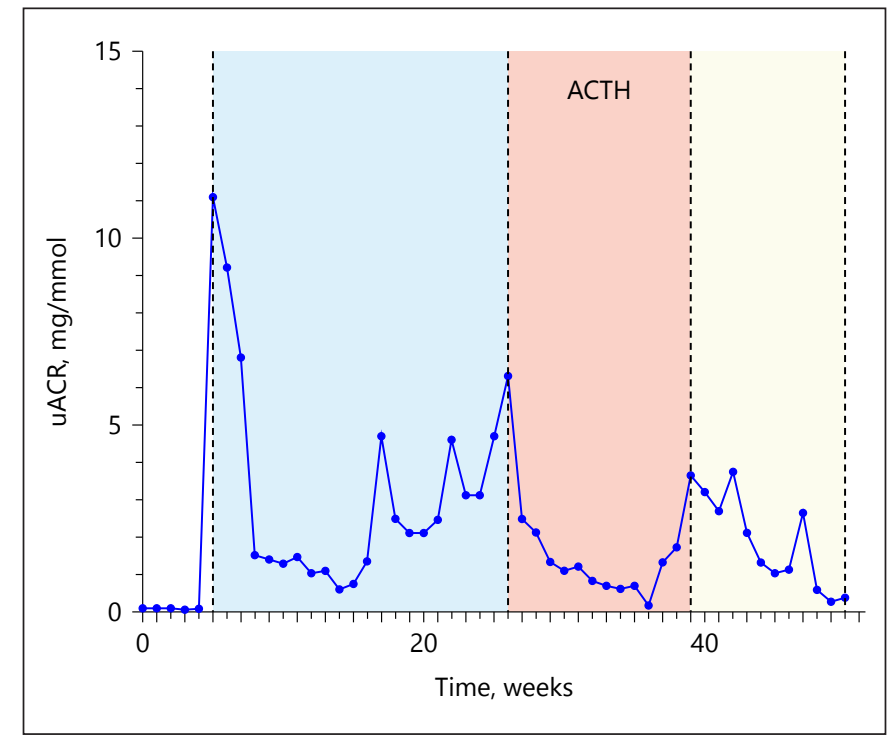

Fig. 2. Time course of the patient's urinary albumin excretion in response to ACTH therapy. UACR at indicated times were collected and plotted. The period of ACTH therapy was colored beige. $\mathrm{ACTH}$, adrenocorticotropic hormone; $\mathrm{uACR}$, urinary albumin to creatinine ratios.

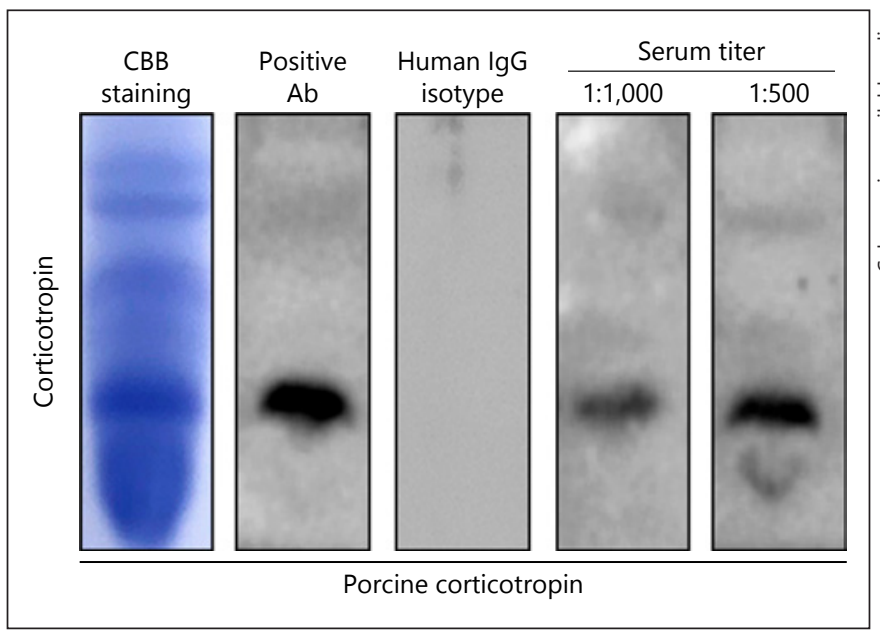

Fig. 3. Immunoblot-based antibody assay reveals the formation of ACTH antibodies in the patient's serum. Standard porcine corticotropin was fractionated on sodium dodecyl sulfate polyacrylamide gel electrophoresis gels, followed by CBB staining (leftist panel). Standard porcine corticotropin was fractionated on sodium dodecyl sulfate polyacrylamide gel electrophoresis gels, transferred to polyvinylidene difluoride membrane, and subjected to immunoblotting overnight with a commercial rabbit $\mathrm{Ab}$ as positive control, control human IgG as negative control, or with the patient's sera (1:500 and 1:1,000 dilution). The blots were then developed by using an anti-rabbit secondary antibody or anti-human IgG antibody. Bands for corticotropin were indicated. CBB, coomassie brilliant blue; $\mathrm{Ab}$, anticorticotropin antibody.
She was initially treated with a high dose $(1 \mathrm{mg} / \mathrm{kg})$ of oral prednisone, ensued by regression of proteinuria. Due to frequent relapses of proteinuria and swelling, she received long-term maintenance therapy with daily $15 \mathrm{mg}$ of oral prednisone, $50 \mathrm{mg}$ twice a day of cyclosporine and $15 \mathrm{mg} /$ day of lisinopril.

The patient responded well to this regimen for approximately 3 years but started noticing substantial adverse effects, including elevated blood pressure and blood glucose levels, truncal obesity, menstrual abnormalities, acne and emotional lability. Dose of glucocorticoid was thus tapered with re-appearance of nephrotic range proteinuria.

She was offered treatment with repository corticotropin of porcine origin (Acthar gel). Treatment regimen of $40 \mathrm{IU}$ ACTH gel as a subcutaneous injection, twice a week, was initiated and her cyclosporine and lisinopril medications were continued in prior dosage. She developed significant remission in proteinuria, regression of swelling, better blood glucose, and generalized feeling of wellbeing on the ACTH gel treatment. Complete remission of proteinuria was noted for about 10 weeks on ACTH, (Fig. 2), after which proteinuria and anasarca re-appeared, and she was re-started on a high dose of glucocorticoid.

At this time, blood was drawn for immunoblot-based antibody assay for detecting the possible development of ACTH antibody. Abundant anti-ACTH antibody was noted in the patient's serum (Fig. 3). Disease relapse was subsequently treated with Rituximab, to which the patient responded completely with disappearance of proteinuria.

\section{Methods and Results}

The patient's blood was collected 3 weeks after relapse of proteinuria, while she was being treated with ACTH gel. Serum was separated and subjected to immunoblot assay as previously described. Standard porcine corticotropin was fractionated on sodium dodecyl sulfate polyacrylamide gel electrophoresis gels and transferred to polyvinylidene difluoride membrane. Subsequently, the membrane was subjected to blocking with Tris-buffered saline containing $5 \%$ bovine serum albumin, and then incubated overnight with either a commercial rabbit anticorticotropin antibody as positive control or with the patient's serum (1:1,000 or 1:500 dilution). Afterwards, the blots were incubated with an anti-rabbit secondary antibody or anti-human IgG antibody. Finally, the blots were washed 3 times with Tris-buffered saline containing $0.1 \%$ Tween 20 and developed with the Enhanced Chemiluminescence Western Blotting Detection System (GE Healthcare) according to the manufacturer's protocols. As a negative control, immunoblots were probed by standard human IgG isotype controls and no bands demonstrated. In stark contrast, immunoblots developed by using the patient's serum evidently revealed abundant IgG antibodies that were reactive to a peptide band also probed by 
the anti-ACTH positive control antibody, suggesting that the patient has high titers of circulating anti-ACTH antibodies. To further ascertain if the anti-ACTH antibody in the patient's serum actually affects the biological functionality of porcine corticotropin, an in vitro test was carried out in B16 melanoma cells, known to express abundant melanocortin receptors. Shown in online supplementary Figure 1 (for all online suppl. material, see www. karger.com/doi/10.1159/000506854), B16 cells were exposed to porcine corticotropin $(100 \mathrm{mIU} / \mathrm{mL})$ in the presence or absence of the patient's serum. Porcine corticotropin treatment apparently triggered the melanocortinergic signaling pathway, as evidenced by an increased phosphorylation of GSK3 $\beta$, a downstream signaling transducer of the melanocortin cascade. This effect was substantially abrogated by addition of the patient's serum, suggesting that the anti-ACTH antibody in the patient's serum is effective in counteracting the biological effect of porcine corticotropin.

Intriguingly, the ACTH antibodies were not associated with any clinical signs of hypoadrenocorticism, or other notable side effects except a delayed-onset resistance to ACTH therapy, entailing that these antibodies are likely specific for porcine ACTH and have negligible cross-reactivity with the patient's native ACTH. This was validated by a separate immunoblot-based antibody assay to verify the specificity of the anti-ACTH antibody in the patient's serum. Shown in online supplementary Figure 2, human ACTH was fractionated by sodium dodecyl sulfate polyacrylamide gel electrophoresis followed by Coomassie Brilliant Blue staining or by immunoblot analysis through incubating the blots overnight with a commercial rabbit anti-human ACTH antibody as positive control or with the patient's serum. The blots were then developed by using an anti-rabbit secondary antibody or anti-human IgG antibody. Despite a clear detection of the human corticotropin by the positive control antibody, no bands were detected by the patient's serum at multiple titers, suggesting that the anti-ACTH antibody in the patient's serum is unlikely reactive to human ACTH.

\section{Discussion}

Corticotropin or ACTH has been successfully used in the treatment of nephrotic syndrome since 1950s. In the past, it was believed that the antiproteinuric effect of ACTH may be attributed to its steroidogenic activity through a direct action on the melanocortin 2 receptor [3,

Relapse of Nephrotic Syndrome after

ACTH Therapy
11] on the adrenal cortex. However, recently more and more studies suggest that ACTH is still quite effective in patients with steroid-resistant glomerular diseases, denoting a steroidogenic-independent proteinurea-reducing activity of ACTH [2]. In consistency, a growing body of evidence suggests that non-steroidogenic melanocortin receptors like MC1R and MC5R are expressed by glomerular podocytes and that the melanocortinergic activity of ACTH conveyed by these melanocortin receptors may contribute to a podocyte protective and antiproteinuric action [3, 12-15].

Structurally, ACTH is a 39 amino acid straight chain polypeptide. It is constituted of the highly conserved $\mathrm{N}$ terminal amino acids $1-24$, and the C-terminal amino acids 25-39 [3]. The first 24 amino acid chain is identical in ACTH derived from all species [10] including human and porcine ACTH. In contrast, the C-terminal amino acids 25-39 of ACTH are species specific and are implicated in antigenicity of the ACTH molecule $[16,17]$. ACTH in the formulation used in our patient is naturally occurring porcine ACTH extracted from porcine pituitary [18] and thus is the complete 39 amino acid chain containing the species-specific 25-39 segment of ACTH. Gelatin in the ACTH gel is a commonly used carrier and unlikely antigenic.

As is common with any biologic medications [6] like insulin or erythropoietin, animal-derived ACTH is antigenic to humans, and antibody formation results in resistance to ACTH therapy $[19,20]$. Even though biologic preparations of porcine origin are thought to be least antigenic, antibodies to ACTH have been well documented in patients with rheumatologic disorders treated by naturally occurring porcine ACTH [7-9]. Most frequently, anti-porcine IgG has been demonstrated against the Cterminal of the polypeptide chain of ACTH; however, there are isolated reports of antibody development to the entire polypeptide chain [16]. There is a case report of antibody formation against short-acting ACTH [10], in which a pediatric patient with steroid-dependent nephrotic syndrome due to MCD was converted to ACTH therapy after developing Cushing syndrome and severe cellulitis. Following a rapid remission of proteinuria, the patient exhibited a delayed-onset resistance to ACTH treatment, concomitant with de novo antibody formation against ACTH. This unusual finding was attributed to the fact that the short-acting ACTH used in this patient was a mixture of pituitary extracts of porcine, bovine, and ovine origins that greatly rendered it antigenic. In consistency, Glass et al. $[8,9]$ have reported that administration of porcine ACTH induces only humoral immunity but no 
cellular component as noted by lack of lymphocyte transformation; however, data in this regard are limited.

In our report, the possibility of antibody reaction to gelatin carrier of the ACTH gel was rejected, as the serum of our patient was strongly reactive to external standard porcine ACTH. We did not come across any similar case report in our extensive literature search, where the initial sustained complete remission of proteinuria induced by ACTH gel treatment was followed by resistance due to antibody development in an adult patient.

\section{Conclusion}

ACTH is an alternative therapeutic modality for refractory proteinuric glomerulopathies like FSGS. However, as quintessential therapeutic biologics, natural ACTH, regardless of purity and origin, is inevitably antigenic in humans and may cause the formation of neutral- izing antibodies in some sensitive patients, ensued by resistance to ACTH therapy. Our findings stress the need to develop ACTH analogues with less immunogenicity for improving its responsiveness in patients with glomerular diseases.

\section{Acknowledgments}

This work was supported in part by the U.S. National Institutes of Health grant DK092485 and DK114006.

\section{Disclosure Statement}

Drs. Rujun Gong and Lance D. Dworkin report research funding from the Mallincrodt Pharmaceuticals, which is not related to this study. Dr. Rujun Gong served as a consultant to the Questcor Pharmaceuticals and the Mallincrodt Pharmaceuticals. All the other authors declared no competing interests.

\section{References}

1 Ponticelli C, Passerini P, Salvadori M, Manno C, Viola BF, Pasquali S, et al. A randomized pilot trial comparing methylprednisolone plus a cytotoxic agent versus synthetic adrenocorticotropic hormone in idiopathic membranous nephropathy. Am J Kidney Dis. 2006 Feb;47(2):233-40.

2 Gong R. The renaissance of corticotropin therapy in proteinuric nephropathies. Nat Rev Nephrol. 2011 Dec;8(2):122-8.

3 Gong R. Leveraging melanocortin pathways to treat glomerular diseases. Adv Chronic Kidney Dis. 2014 Mar;21(2):134-51.

4 Hogan J, Bomback AS, Mehta K, Canetta PA, Rao MK, Appel GB, et al. Treatment of idiopathic FSGS with adrenocorticotropic hormone gel. Clin J Am Soc Nephrol. 2013 Dec; 8(12):2072-81.

5 Hladunewich MA, Cattran D, Beck LH, Odutayo A, Sethi S, Ayalon R, et al. A pilot study to determine the dose and effectiveness of adrenocorticotropic hormone (H.P. Acthar ${ }^{\circledR} \mathrm{Gel}$ ) in nephrotic syndrome due to idiopathic membranous nephropathy. Nephrol Dial Transplant. 2014 Aug;29(8): 1570-7.

6 Garcês S, Demengeot J. The Immunogenicity of Biologic Therapies. Curr Probl Dermatol. 2018;53:37-48.
7 Landon J, Friedman M, Greenwood FC. Antibodies to corticotrophin and their relation to adrenal function in children receiving corticotrophin therapy. Lancet. 1967 Mar; 1(7491): 652-5.

8 Glass D, Daly JR. Development of antibodies during long-term therapy with corticotrophin in rheumatoid arthritis. I. Porcine ACTH. Ann Rheum Dis. 1971 Nov;30(6):589-92.

9 Glass D, Nuki G, Daly JR. Development of antibodies during long-term therapy with corticotrophin in rheumatoid arthritis. II. Zinc tetracosactrin (Depot Synacthen). Ann Rheum Dis. 1971 Nov;30(6):593-6.

10 Wang P, Zhang Y, Wang Y, Brem AS, Liu Z, Gong R. Acquired Resistance to Corticotropin Therapy in Nephrotic Syndrome: Role of De Novo Neutralizing Antibody. Pediatrics. 2017 Jul;140(1):e20162169.

11 Dores RM. Adrenocorticotropic hormone, melanocyte-stimulating hormone, and the melanocortin receptors: revisiting the work of Robert Schwyzer: a thirty-year retrospective. Ann N Y Acad Sci. 2009 Apr;1163(1):93-100.

12 Chhajlani V. Distribution of cDNA for melanocortin receptor subtypes in human tissues. Biochem Mol Biol Int. 1996 Feb;38(1):73-80.

13 Voisey J, Carroll L, van Daal A. Melanocortins and their receptors and antagonists. Curr Drug Targets. 2003 Oct;4(7):586-97.
14 Cone RD. Studies on the physiological functions of the melanocortin system. Endocr Rev. 2006 Dec;27(7):736-49.

15 Sánchez E, Rubio VC, Cerdá-Reverter JM. Characterization of the sea bass melanocortin 5 receptor: a putative role in hepatic lipid metabolism. J Exp Biol. 2009 Dec;212(Pt 23): 3901-10.

16 Fleischer N, Abe K, Liddle GW, Orth DN, Nicholson WE. ACTH antibodies in patients receiving depot porcine $\mathrm{ACTH}$ to hasten recovery from pituitary-adrenal suppression. J Clin Invest. $1967 \mathrm{Feb}$;6(2):196-204.

17 Imura H, Sparks LL, Tosaka M, Hane S, Grodsky GM, Forsham PH. Immunologic studies of adrenocorticotropic hormone (ACTH): effect of carboxypeptidase digestion on biologic and immunologic activities. J Clin Endocrinol Metab. 1967 Jan;27(1):15-21.

18 Gettig J, Cummings JP, Matuszewski K. H.p. Acthar gel and cosyntropin review: clinical and financial implications. P T. 2009 May; 34(5):250-7.

19 Rosenberg AS. Immunogenicity of biological therapeutics: a hierarchy of concerns. Dev Biol (Basel). 2003;112:15-21.

20 Schellekens $\mathrm{H}$. The immunogenicity of therapeutic proteins. Discov Med. 2010 Jun;9(49): $560-4$. 